\title{
Deck the walls with anisotropic colloids in nematic liquid crystals
}

Yimin Luo, ${ }^{\dagger}$ Tianyi Yao, ${ }^{\ddagger}$ Daniel A. Beller, ${ }^{\llbracket}$ Francesca Serra, ${ }^{\S}$ and Kathleen J.

\author{
Stebe $*,+, \|$ \\ $\dagger$ Department of Chemical and Biomolecular Engineering, University of Delaware, Newark \\ $\ddagger$ Department of Chemical and Biomolecular Engineering, University of Pennsylvania, \\ Philadelphia \\ \Department of Physics, University of California, Merced \\ $\S$ Department of Physics and Astronomy, Johns Hopkins University, Baltimore \\ ||Kathleen Stebe was a 2018 Langmuir lecturer and presented the content of this article \\ E-mail: kstebe@seas.upenn.edu
}

Supplemental Note 1 The anchoring strength in simulation are set so that the ratio of extrapolation length to correlation length stays the same. More specifically, using the simulation values, $\xi=L / W \approx 0.8 \mathrm{~nm}$ for the homeotropic anchoring conditions which can also be expressed as the ratio of the non-dimensionalized values in units of the lattice spacing, $\xi=L / W \approx(2.32 / 12.9) \Delta x \approx 0.8 \mathrm{~nm}$ with $\Delta x=4.5 \mathrm{~nm}$. The simulated colloid diameter $2 \mathrm{a}=100 \mathrm{~nm}$ is thus about 100 times greater than $\xi$. In experiments, the colloid diameter is $2 a \approx 12 \mu \mathrm{m}$ while $\xi=L / W \approx 0.1-1 \mu \mathrm{m}$ for anchoring strengths in the range $10^{-4}-10^{-5} \mathrm{~J} \mathrm{~m}^{-2}{ }^{1}$ giving a ratio $2 a / \xi \approx 10-100$.

Supplemental Note 2 To explore relationship between colloid aspect ratio and entrance length, we specifically pick ellipsoids whose minor axis $2 \beta$ is less than the well-opening. While it is more intuitive to plot the entrance length with respect to $\beta$, normalized by the entrance 
radius $R_{\text {ent }}$, this plot is not as predictive for the fate of whether the ellipsoid or sphere will enter (Fig. S8). We notice that the sign of $L$ seems to be independent of $\beta / R_{\text {ent }}$. While a tight opening is expected to play a role in restricting the entering of the ellipsoid, the splay field is expected to play an even stronger role. Dipoles are analogous to Saturn I in their diverging splay field. This field is stronger for particles of higher $k$, which needs to equilibrate lower inside the tapered well in order to be stabilized. This is compatible with our previous observation that dipoles sit lower in a well compared to Saturn rings to better match their splay deformation.

\section{Supplemental Figures}
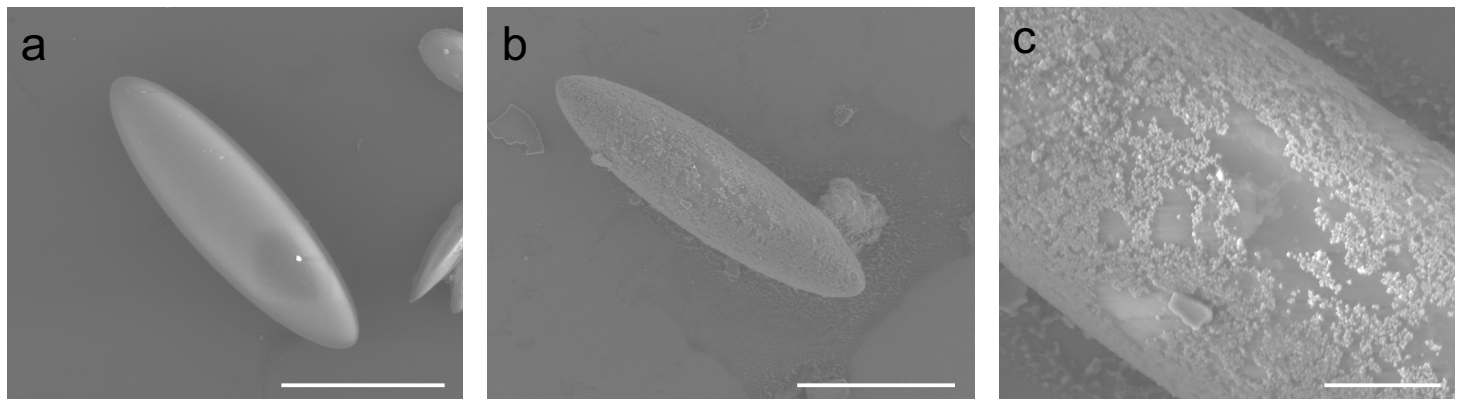

Figure S1: Scanning electron microscopy of the particles used in this study. (a) Stretched polystyrene particles before coating (b) after coating and zoomed in view of the particle surface in (c). The scale bars are $25 \mu \mathrm{m}$ in (a-b) and $5 \mu \mathrm{m}$ in (c).
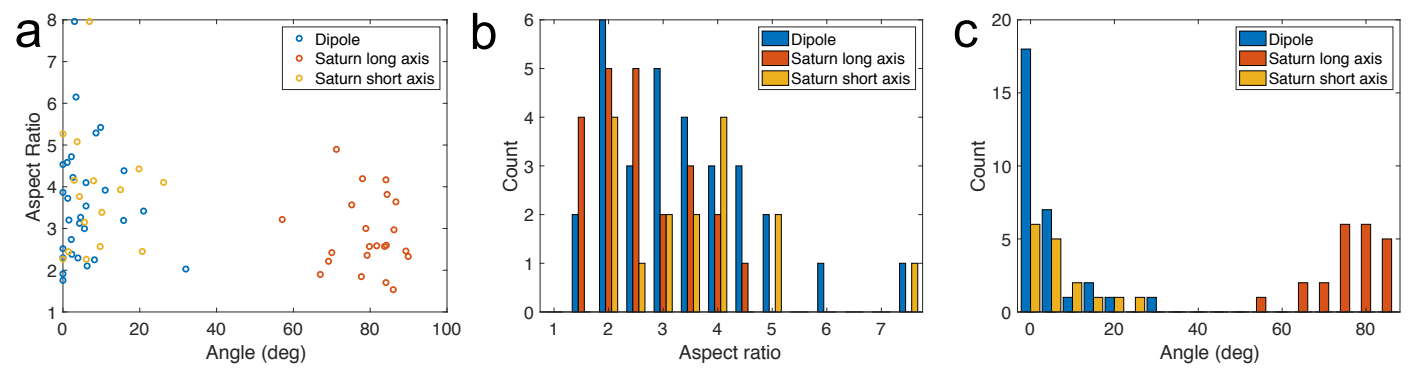

Figure S2: (a) Scatter polar of aspect ratio $k$ versus colloid orientation $\theta$. (b-c) Histograms of (b) aspect ratios and (c) orientations of colloids of different defects. 

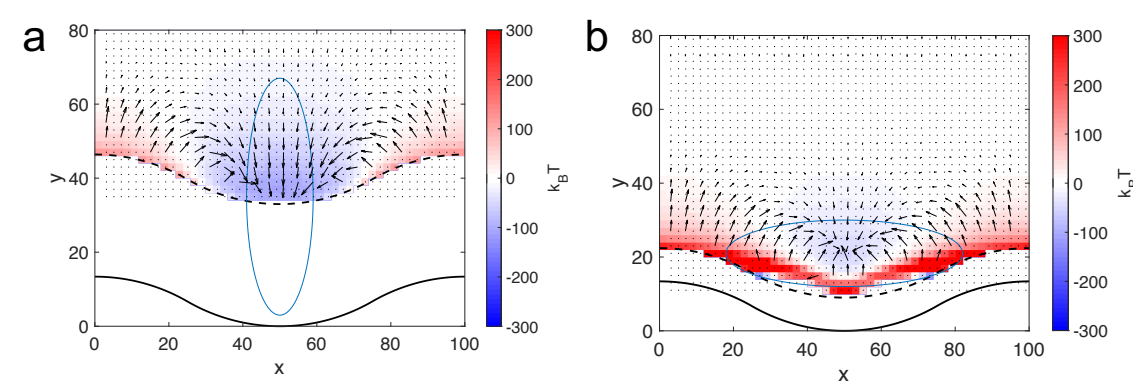

Figure S3: (a-b) Simulations of total energy of the ellipsoid (long axis $a=143 \mathrm{~nm}$, and short axis $b=36 \mathrm{~nm}$ ) when the center of mass of the particle is located at various locations above the wells for (a) Saturn I and (b) Saturn II. Blue ellipses denote the position the ellipsoid when the system is at lowest energy state. Solid line denotes the wall. Dashed line denotes excluded region next to the wall. Arrows denote the gradient, which is the force the ellipsoid experiences assuming no rotation occurs.
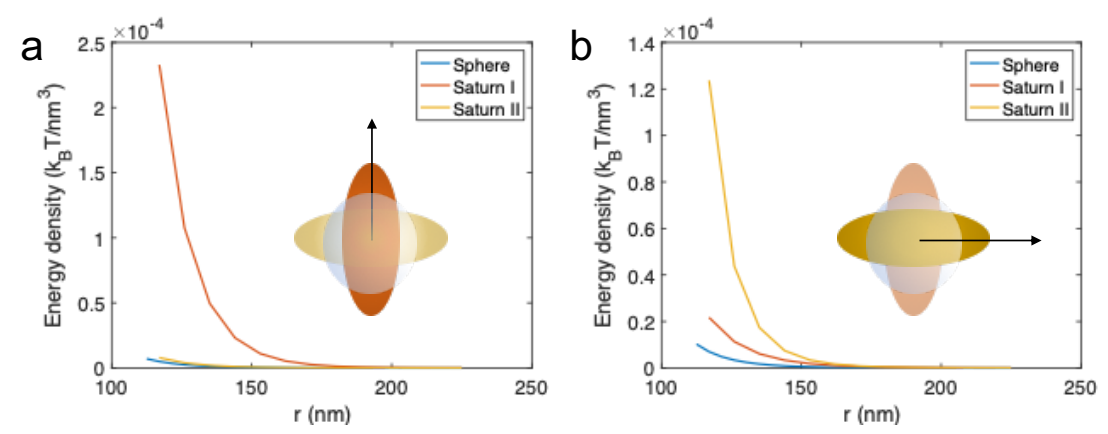

Figure S4: Energy density decay away from Saturn I, Saturn II $(k=2)$ and a sphere of equal volume is plotted against distance to center of mass $r$ along azimuthal and equatorial directions to assess the rate of decay of distortion with distance from these colloids. (a) Energy decay along the azimuthal direction. (b) Energy decay along the equatorial position. Simulation dimensions: $2 a=112.5 \mathrm{~nm}, \alpha=90 \mathrm{~nm}$, and $\beta=45 \mathrm{~nm}$.
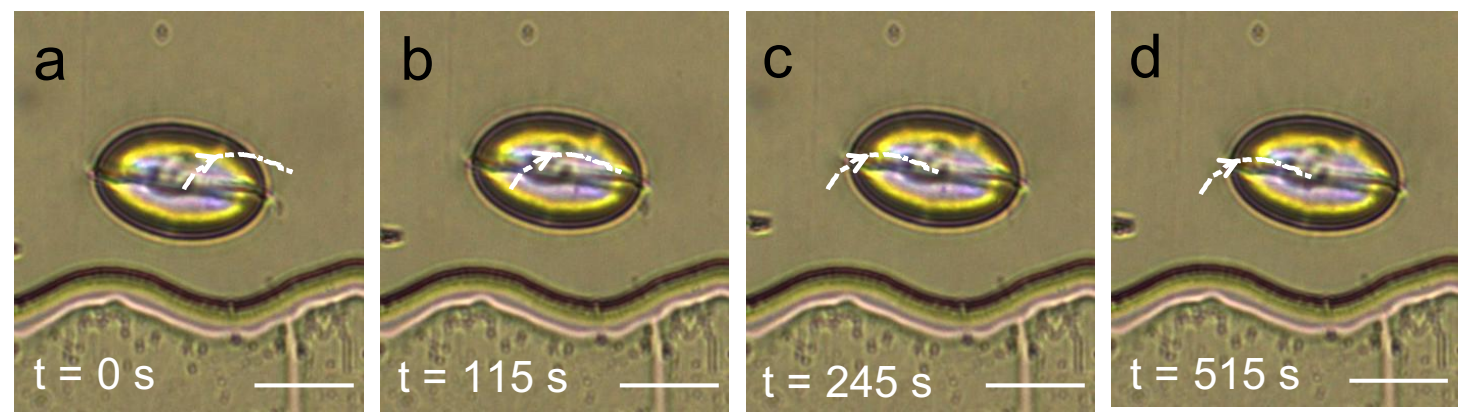

Figure S5: Time lapse images of a Saturn II migrating horizontally, to above the well without docking. The scale bar is $25 \mu \mathrm{m}$. 

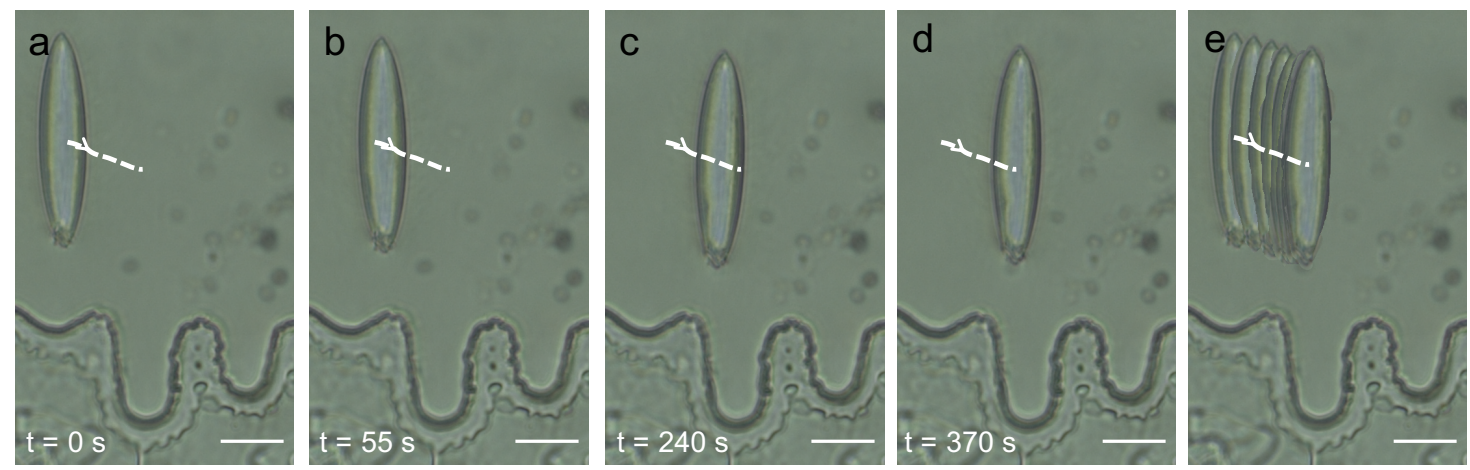

Figure S6: Time lapse images of a dipole with defect oriented downwards migrating but does not enter a deep well. The scale bar is $25 \mu \mathrm{m}$.
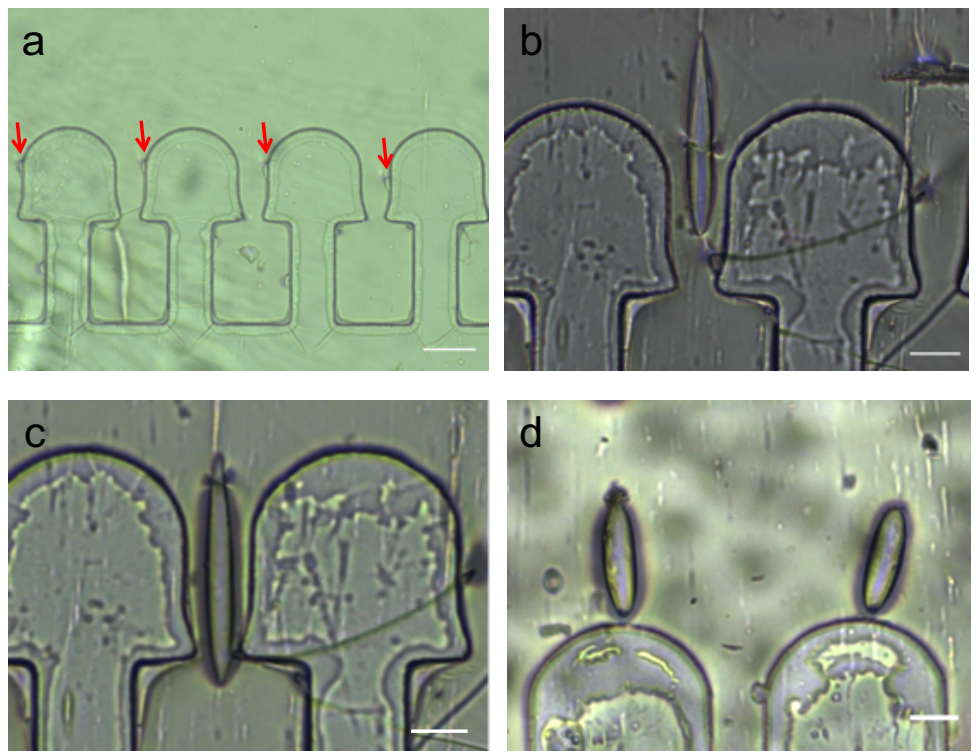

Figure S7: (a) Defects encircle the feature for a cell of thickness $T=21 \mu \mathrm{m}$. Because of the presence of many corners, it is easy for a defect to form and entangle with the structure. (bc) Colloids with Saturn ring defect interact with the defect line on the feature. (d) Colloids with dipoles oriented downwards dock on the cap portion of the geometry. The scale bar is $100 \mu \mathrm{m}$ in (a) and $25 \mu \mathrm{m}$ in (b-d). 


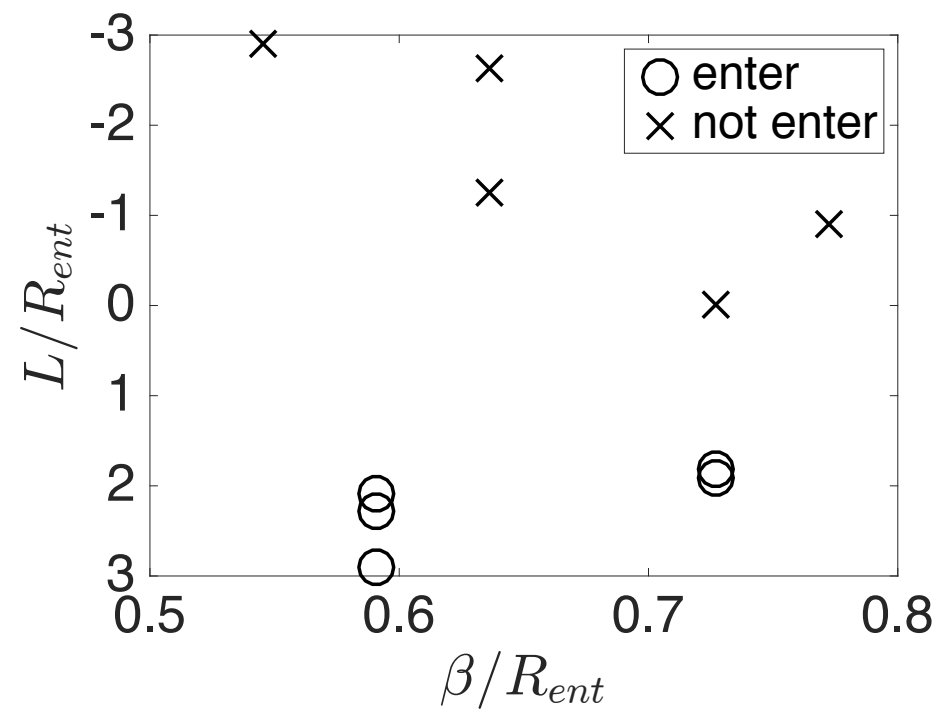

Figure S8: Entrance length $L$ plotted against minor axis $\beta$ of the ellipsoids for mushroomtype wells, normalized by the entrance radius $R_{\text {ent }}$. 


\section{Supplemental Videos}

- Supplemental Video 1: A vertical ellipsoid $\left(\theta=0^{\circ}\right)$ docks inside a shallow well. The frame rate is $0.2 \mathrm{fps}$. The video is at $50 \times$ real time. The duration of the video is 750 S.

- Supplemental Video 2: A horizontal ellipsoid $\left(\theta=90^{\circ}\right)$ migrates towards the centerline of a shallow well, finding its equilibrium position. The frame rate is $0.2 \mathrm{fps}$. The video is at $50 \times$ real time. The duration of the video is $975 \mathrm{~s}$.

- Supplemental Video 3: An ellipsoid first migrates towards the center line, then rapidly insert into a deep well. The frame rate is $0.2 \mathrm{fps}$. The video is at $50 \times$ real time. The duration of the video is $725 \mathrm{~s}$.

- Supplemental Video 4: A sphere $(k=1)$ enters the through-pore geometry and reaching its final equilibrium position. The frame rate is $0.2 \mathrm{fps}$. The video is at $40 \times$ real time. The duration of the video is $475 \mathrm{~s}$.

- Supplemental Video 5: An ellipsoid $(k=2)$ enters the through-pore geometry and reaching its final equilibrium position. The frame rate is $0.2 \mathrm{fps}$. The video is at $50 \times$ real time. The duration of the video is $260 \mathrm{~s}$.

- Supplemental Video 6: An ellipsoid $(k=4)$ enters the through-pore geometry and reaching its final equilibrium position. The frame rate is $0.2 \mathrm{fps}$. The video is at $50 \times$ real time. The duration of the video is $400 \mathrm{~s}$.

\section{References}

(1) Kočevar, K.; Muševič, I. Surface-induced nematic and smectic order at a liquid-crystalsilanated-glass interface observed by atomic force spectroscopy and Brewster angle ellipsometry. Phys. Rev. E 2002, 65, 021703. 